\title{
Value-Added Services in Hospitals: Does Chiropractic Care Have Economic and Marketing Impact
}

\section{Szydlowski $\mathrm{SJ}^{1^{*}}$ and Linhares $\mathrm{DM}^{2}$}

${ }^{1}$ University of Scranton, 800 Linden St, Scranton, PA, USA

${ }^{2}$ College of Graduate and Continuing Education, University of Scranton, 800 Linden St, Scranton, PA, USA

"Corresponding author: Steven J Szydlowski, Master of Health Administration Program Director/Professor, University of Scranton, 800 Linden St, Scranton, PA, USA, Tel: (570) 941-4367; E-mail: steven.szydlowski@scranton.edu

Received date: February 11, 2016; Accepted date: March 01, 2016; Published date: March 08, 2016

Copyright: @ 2016 Szydlowski SJ, et al. This is an open-access article distributed under the terms of the Creative Commons Attribution License, which permits unrestricted use, distribution, and reproduction in any medium, provided the original author and source are credited.

\begin{abstract}
Chiropractic services as mainstream medicine continues to be a major debate among allopathic providers, chiropractic physicians, payers, consumers, and most health care stakeholders. It has been viewed by many as an alternative to traditional treatment for pain relief, structural manipulation for body alignment, and other acute and chronic anatomy and physiological conditions. Historically viewed by many as the outskirts of medical care, chiropractic care recently has become more prevalent among first and second choice care among consumers for certain health ailments. The evolution of complementary and alternative medicine (CAM) beginning in the late 1990's has helped support the chiropractic profession in establishing ground to be more mainstream medicine including some claims coverage by insurance carriers. Post Patient Protection and Affordable Care Act (PPACA) America has one of its main focuses on improving primary and preventive care as well as hospital care across the United States with a purpose of reducing the overall cost of healthcare. Chiropractic care, being one of the largest CAM approaches, is certain to see changes. In this systemic review article $(N=22)$ we will look at the role of chiropractic services in hospital settings, its funding, and potential marketing and economic value added to hospitals and the health system. The authors identify relevant literature to the research question through an exhaustive review and draw conclusions based on common factors within the literature. Hospital continues to find treatment protocols and efficiencies to reduce cost per inpatient stay. The results of this study suggest that chiropractic care can reduce cost per stay and improve health outcomes. The impact of increased integration of chiropractic services into hospital settings needs to be studied to determine long-term cost/benefit.
\end{abstract}

Keywords: Health economics; Health care; Care quality; Outcome research

\section{Introduction}

The National Center for Complementary and Alternative Medicine (NCCAM) describes complementary and alternative medicine (CAM) as a non-mainstream approach to health care that is used together with or in place of conventional medicine, be it via the use natural products or through mind and body practices [1]. Out of those who opt for these alternative services evidence shows that natural products, deep breathing exercises, meditation, chiropractic and osteopathic care, massage therapy, and yoga rank as the top modalities used by consumers of CAM [2]. The most recent data on CAM expenditures suggests that in 2007 adults in the United States had spent approximately $\$ 33.9$ billion on CAM related therapies; about twothirds of the total ( $\$ 22.0$ billion) was expended on CAM products, classes and materials and the other one-third (\$11.9 billion) was expended on practitioner visits alone $[3,4]$, recently demonstrated that integrative health service can save between $\$ 156$ - $\$ 431$ per day for oncology and radiation patients. These authors discuss further cost reductions in both inpatient and outpatient service lines.

With the passing of the Patient Protection and Affordable Care Act (PPACA), enacted by congress in 2010, a brief mention concerning provider discrimination has left CAM practitioners with some optimism for the future. In Section 2706 of the PPACA [5], titled Nondiscrimination in Health Care, it is stated that, "A group health plan and a health insurance issuer offering group or individual health insurance coverage shall not discriminate with respect to participation under the plan or coverage against any health care provider who is acting within the scope of that provider's license or certification under applicable State law." Organizations like the American Chiropractic Association have long since supported the non-discrimination provision and have argued for both the inclusion of Doctors of Chiropractic as recognized providers and for the U.S. Department of Health and Human Services' evaluation of all state's Essential Health Benefits (EHB) benchmark plans to ensure non-discrimination [6]. Chiropractic care is one of the most utilized CAM modalities and accounts for approximately three quarters of the overall CAM market. Data suggests that its usage has increased from 11.5 million adult users in 2002 to 11.9 million adult users in 2008 and estimates of inflationadjusted national expenditure shows an 11 percent increase, from $\$ 6.2$ billion in 2002 to $\$ 6.9$ billion in 2008 [7]. Literature points to a slow process of fully integrating CAM into the U.S. health care system. Impeding factors include lack of scientific evidence on safety and prioritization of research on lesser used modalities, consumer assumption that some CAM therapies are more natural thus safe, conventional medical providers lacking education about successful CAM therapies, and lastly lack of communication between patient and physician about their use of CAM [8], Interestingly, though the described factors impede the quick advancement of CAM, hospitals nonetheless are utilizing these various CAM modalities in both inpatient and outpatient settings. 
The PPACA's push for safer and more cost effective health care is changing the playing field for CAM practitioners and various stakeholders alike. Our interest in analyzing chiropractic services in hospital settings lies in the potential clinical benefit and the consumerism value added to the system. We shall examine other implications of the PPACA that may affect CAM and discuss organizations that are making an effort to support CAM research and education.

\section{Methodology}

A comprehensive review of the literature and published reports was conducted to assess the prevalence and role chiropractic care has in hospital settings $(\mathrm{N}=22)$. A systemic review of the literature was conducted to identify themes and trends regarding chiropractic services. The articles selected in the review included: cost / benefit analysis in acute settings, primary care settings, and community-based settings to establish efficacy and effectiveness, economic evaluation, contribution as ancillary service, and consumerism. The review timeframe consisted of articles published between 2005 - 2015. Search terms related to chiropractic services related to the topics identified above. Findings were condensed into common categories and summaries were provided.

\section{Results}

\section{Clinical and cost benefit}

Current CAM acceptability can be credited to its growing use, the establishment of the NCCAM at the National Institute of Health (NIH), growing scientific investigation of CAM's safety and efficacy, and lastly physicians receiving education about CAM in order to motivate both interest and its acceptance [9]. CAM therapies must have a continuous backing of scientific data in order for continued acceptance and eminent success. Such data must demonstrate its clinical value as well as its role in cost reduction in order to gain favour from its opponents.

To discuss all CAM modalities in detail and their current scientific backing, or lack thereof, would be an exhaustive undertaking and is not the defined goal of this paper. Nonetheless, it is important to discuss data showing CAM's benefits and disadvantages that are presently available. As possible stakeholders it is imperative to recognize that data on CAM efficacy and economic evaluations suggest conclusions specific to the settings and potential treatment of health conditions described in the analyzed studies.

From a clinical perspective, a recent review of 270 studies has concluded that some CAM therapies tended to reduce pain more so than usual medical care, physical therapy, or no treatment. Let's take for instance acupuncture, which proved to be more effective when compared to no treatment in improving pain intensity, disability, functioning, well-being, and range of mobility, immediately after the treatment. Other acupuncture trials included the modality being used to treat chronic nonspecific low back pain resulting in significantly lower pain intensity than placebo if administered immediately posttreatment; but no different from placebo if done perfunctorily posttreatment [10]. The analysis also determined that for both lower back and neck pain, spinal manipulation was significantly better than placebo or no treatment in reducing pain immediately or short-term after the end of treatment. Spinal manipulation was said to be better than acupuncture in improving pain and function in chronic nonspecific low back pain. Out of the literature analyzed by the author a few conclusive points regarding the literature have been made. The major objections seem to be more so directed at the actual quality of study and lack in diversity of the conditions treated by the studied modalities rather than the effectiveness of the modality itself. Additionally, chiropractors serving the role of primary specialist in health problems such as: spine disorder has drawn interest in Canada and other countries to meet societal needs [11].

A systematic review of economic evaluations, those measuring cost alongside safety and effectiveness, of CAM therapies concluded that out of the 56 comparisons made 16 (29\%) were actually cost saving; the researched therapy had better health outcomes and lower costs than usual care alone [12]. The researched modalities included therapies such as acupuncture, certain manual therapy treatments (including chiropractic care), some natural products and others such as Tai chi. conclusively; various CAM modalities have proven to be clinically effective and cost saving for numerous health conditions.

\section{Consumerism in hospitals}

CAM began to gain popularity in the 1960's. In the 1990's surveys identified a larger population of users; those who despite their frustrations with conventional medicine chose to complement their care with CAM. As the years progressed CAM use and expenditures grew leading to the need of research to test its safety and efficacy which then led to the formation of the Office of Alternative Medicine, later to be changed to the NCCAM under the NIH [9]. Data suggests that back in 2007 over one third of Americans had used some form of CAM therapy and a different source finds visits to an alternative care provider from 2002 to 2008 had increased from 15.2 million to 16.1 million (6\%) respectively [2,7]. CAM has also been the focus of physicians and medical schools with surveys showing over $50 \%$ medical school faculty approval of modalities such as acupuncture and chiropractic care seen as legitimate medical practices and through the offering of electives in CAM as part of their medical education programs [9].

Through increased acceptance and use CAM has made its way in hospital settings. The most current data on hospitals and their use of CAM, derived from the 2011 American Hospital Association (AHA) Annual Survey, established that out of 4,782 reporting hospitals 119 (2.5\%) of them offered chiropractic services and 902 (18.9\%) offered CAM services, services including acupuncture, chiropractic, homeopathy, osteopathy, diet and lifestyle changes, herbal medicine, massage therapy, etc. [13]. As our prior arguments indicate CAM research is still in its infancy and various stakeholders recognize the need for more long-term / quality data on its efficacy. But why then have hospitals already begun to integrate CAM programs? The simple answer: demand.

The 2010 Complementary and Alternative Medicine Survey of Hospitals sought out to analyze information about CAM programs and services offered by hospitals. Out of interest to us are first reasons why these hospitals offered CAM services and few financial implications. It was said that patient demand, clinical effectives, and reflection of organization's mission were the top three main reasons why the hospitals chose to offer care and their evaluation on the success of the offered therapies were highly based on patient satisfaction feedback [14]. A second noteworthy finding concluded that payments for CAM services were predominantly patient self-pay, followed by no charge (philanthropic coverage or included as part of overall care), and thirdparty reimbursement [14]. Nationally, most CAM services in 
ambulatory settings are paid out-pocket; which may explain the lack of more substantial growth in expenditure in the past years [7]. With this in mind and current insurance changes mandated by the PPACA we are bound to see fluctuations in the CAM market.

Literature showing recommendations and challenges of CAM integration in hospitals is becoming gradually more available. For example, evidence of an integrative medicine program which started in 2006 at Beaumont Cancer Institute in Royal Oak, MI, credits the demand for CAM services and its ability to increase patient satisfaction, if done successfully, as two of the many reasons to consider a venture in integrating CAM in hospital settings. The program initially adopted massage therapy and later, with continued success, grew to include 11 different types of CAM therapies. Success was credited to educating staff and administration to CAM, staffing experienced CAM practitioners, marketing the program, and having a comprehensive business plan to account for any possible hardships / liabilities [15]. A number of other articles emphasize leadership as a guide to effective implementation of a successful CAM program. The appointment of what are called "champions," or passionate and visible leaders of the programs, is said to be a critical factor for success along with employing the $[16,17]$.

\section{Healthcare law}

The PPACA ensures that every state offer health insurance policies, both inside and outside of the Health Insurance Marketplace, as comprehensive services called EHB. HealthCare.gov states that starting as of 2014 plans must include these services, which vary state to state [18]. Our focus was on CAM services listed on each state's EHB benchmark plan. We analyzed data listed under The Center for Consumer Information and Insurance Oversight (CCIIO) of the Center for Medicare and Medicaid Services (CMS). In Figure 1 we see that out of 50 states 45 cover chiropractic services under the state's EHB and 5 states cover acupuncture (some states offer coverage for both modalities). There are a total of 4 states that don't cover any CAM modality. As mandated by the PPACA we see that a total of 90 percent of states will now cover chiropractic care to some extent as defined by the state's EHB benchmark plans. The amount of coverage differs from state to state and ranges from visits per year, to a dollar amount per year, to having a no limit designation in a few states. From a public policy standpoint, enacting initiatives to facilitate the use of chiropractic involvement in public health may support social ecological aspects of creating health communities impacting safety, health through the lifespan, and city infrastructure planning [19].

\section{Marketing and economic implications}

Is hospital inclusion of chiropractic services a reactive approach to the market or a proactive attempt to provide additional service lines to generate revenue? Based on the literature review above, the available information suggests that an inclusion of chiropractic services in hospital settings is merely a reactive approach to customer demand for those services. Critisism from the allopathic community is often an obstacle to true integration of chiropractic services in hospital settings. The author concludes from personal experience as a chief executive officer for an integrative medicine clinic for fourteen years that patients most often end up in the chiropractic exam room when conventional therapies and pain relieving prescriptions are no longer wanted and consumers seek out other alternatives to pain management and structural ailments (Figure 1).

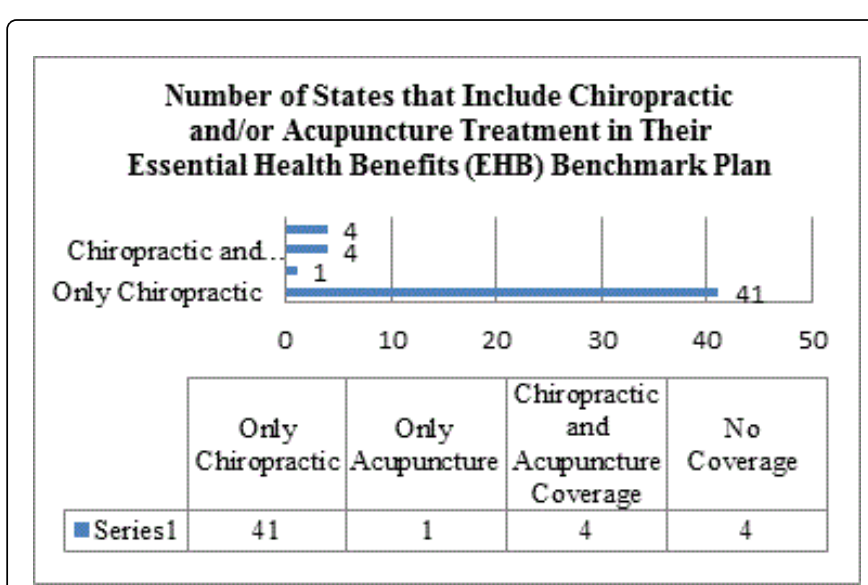

Figure 1: Author's analysis of Complementary and Alternative Medicine (CAM) coverage in states' Essential Health Benefits (EHB) Benchmark Plans as indicated by The Center for Consumer Information and Insurance Oversight (CCIIO) of the Center for Medicare and Medicaid Services (CMS) [20].

Operational and economic benefits have been realized through chiropractic services in health settings. Some efficiencies and information enhancement for patient care were present in the use of electronic medical records which is yet to be quantified [21]. Chiropractic care appeared to be cost effective in treating chronic and acute conditions related to low back pain that can quantify further savings in hospitals $[22,23]$.

Can and should hospital administrators view chiropractic services and CAM as an opportunity to offer a new service line or ancillary service as a more proactive, intentional business practice? The question continues to revolve around medical staff buy-in and implications on other medical services volume that still serve as the core business units for hospitals. Additionally, the level of insurance coverage by providers continues to vary among health plans, although increased coverage has been the trend. With the buy-in from key hospital stakeholders, chiropractic services and CAM may position a hospital as having value-added to the many consumers and billion dollar industry that pays out-of-pocket for those services. Given the geographic location and demographics of a population, these services may be more appealing to health care leaders and planners as competition intensifies.

\section{Discussion and Conclusion}

The healthcare environment is changing and CAM is part of that change. With the increase in CAM research top modalities are bound to see consumer utilization and healthcare approval fluctuation. Healthcare managers in areas subject to high demand of CAM therapies will be faced with the decision of whether or not to model their organization based on this external demand for alternative or complementary treatment. It is important to note that on the provider side the new law does not set reimbursement rates for CAM care. Contrary to the provider side, consumers of these new health plans must be aware of the available providers that make up the current insurance network and restrictions to use. The EHB is simply a model for the fundamental benefits offered by insurance companies in a state and such companies can choose to modify / increase CAM coverage. 
Hospital providers may find alternative and complementary therapies as ancillary service lines that can produce market value for consumers seeking those types of services. As evidenced by the significant amount of out-of-pocket payments for CAM services by consumers, in particular chiropractic services, hospitals may be at a market disadvantage as consumer demand continues to grow. Hospitals and other health care providers can use chiropractic services to enhance revenue, meet consumer demands, and ultimately improve quality of care using a more comprehensive-approach.

From a pure financial perspective, chiropractic service setting requires limited space within a facility. The service is generally provided in less than twenty minutes similar to a primary care physician visit. Therefore, the patient turnover can result in four to five patients per chiropractic table per hour. Within the context of a larger health system, executives may consider locating chiropractic services in an outpatient location with other service lines. The demanded service by consumers can generate ancillary revenue at a relatively low cost per visit. An on-going challenge remains for health executives contemplating the integration of chiropractic services in a hospital or on a hospital campus. That is the continued debate between the efficacies of chiropractic services by many allopathic providers. Although plenty of research and consumer buying behaviour support the value of chiropractic care and CAM, resistance is still present by many allopathic providers. It will be critical for open communication and deep dialogue to between the various stakeholders to clearly understand the role of chiropractic services for a hospital. It is important to note that acceptance and adoption of chiropractic service and CAM modalities may vary based on geography and culture within parts of the country.

\section{References}

1. National Center for Complementary and Alternative Medicine (2008) Complementary, Alternative, Integrative Health: What's In a Name?

2. Barnes PM, Bloom B, Nahin RL (2008) Complementary and alternative medicine use among adults and children: United States 2007. National health statistics reports; no 12. Hyattsville MD: National Center for Health Statistics.

3. Nahin RL, Barnes PM, Stussman BJ, Bloom B (2009) Costs of Complementary and alternative medicine (CAM) and frequency of visits to CAM practitioners: United States, 2007. National health statistics reports; no 18. Hyattsville MD: National Center for Health Statistics.

4. Russo R, Diener I, Stitcher M (2015) The low risk and high return of integrative health services. Healthcare Financial Management 11: $114-120$.
5. Patient Protection and Affordable Care Act (2010).

6. American Chiropractic Association (2012) ACA Presses HHS on NonDiscrimination Enforcement.

7. Davis MA, Martin BI, Coulter ID, Weeks WB (2013) US Spending on Complementary and Alternative Medicine During 2002-08 Plateaued, Suggesting Role in Reformed Health System. Health Affairs 32: 45-52.

8. Nahin RL, Pontzer CH, Chesney MA (2005) Racing towards the Integration of Complementary and Alternative Medicine: A Marathon or a Sprint. Health Affairs 24: 991-993.

9. Ruggie M (2005) Mainstreaming Complementary Therapies: New Directions in Health Care. Health Affairs 24: 980-990.

10. Furlan A, Yazdi F, Tsertsvadze A, Gross A, Van Tulder M, et al. (2010) Complementary and Alternative Therapies for Back Pain II.

11. Erwin WM, Korpela AP, Jones RC (2013) Chiropractors as primary spine care providers: Precendents and essential measures. The Journal of the Canadian Chiropractic Associatio 57: 285-291.

12. Herman PM, Poindexter BL, Witt CM (2012) Are complementary therapies and integrative care cost-effective? A systematic review of economic evaluations. BJM Open 2: e001046.

13. AHA hospital statistics (2012) Chicago.

14. Ananth S (2010) Complementary and alternative medicine survey of hospitals. Alexandria VA: Samueli Institute.

15. Lanni T, Patricolo GE (2013) The business of integrative medicine in a large hospital system. Journal of Hospital Administration 3.

16. Boon HS, Kachan N (2008) Integrative medicine: A tale of two clinics. BMC Complementary and Alternative Medicine 8: 32.

17. Szydlowski SJ, Amato PP (2010) Business Strategies Applied to an Integrative Medicine Practice: A Case Study on Sustainability. Integrative Medicine: A Clinician's Journal 9: 28-32.

18. Glossary: Essential Health Benefits (2014) HealthCare gov.

19. Johnson C (2012) Chiropractic care and public health: Answering difficult questions about safety, care through the lifespan, and community action. Journal of Manipulative and Physiological Therapeutics 35: 493-513.

20. Additional Information on Proposed State Essential Health Benefits Benchmark Plans (2014) Centers for Medicare \& Medicaid Services.

21. Elder C (2015) Acupuncture and chiropractic care: Utilization and electronic medical record capture. American Journal of Management 21: $414-421$.

22. Blanchette M, Bussieres A, Stochkendahl MJ, Boruff J, Harrison P (2015) Effectiveness and economic evaluation of chiropractic care for the treatment of low back pain: A systematic review protocol. Systematic Reviews Journal 4.

23. Hass M, Sharma R, Stano M (2005) Cost-effectiveness of medical and chiropractic care for acute and chronic low back pain. Journal of Manipulative and Physiological Therapeutics 28: 555-563. 